\title{
NUMERICAL INVESTIGATION OF FLOW AND HEAT TRANSFER IN COMMUNICATING CONVERGING AND DIVERGING CHANNELS
}

\author{
M. Tahir Erdinç1,*, T. Yılmaz²
}

\begin{abstract}
Fluid flow and heat transfer in communicating converging and diverging channel has been numerically investigated. Channels are assumed to be at constant wall temperature and the flow is assumed to be steady state and incompressible. Since the flow and temperature fields to repeat periodically after a certain developing length, periodic boundary conditions are used for the calculations. Finite volume method is used to solve the governing differential equations numerically. Computations are performed for different values of the plate angles and Reynolds numbers. Moreover, velocity distributions along the flow field are illustrated. It was found that the converging- diverging channels destroy the boundary layer significantly and Nusselt number is found to be about $400 \%$ higher than those of parallel plate channels, whereas due to vortex formation, pressure drop increases also.
\end{abstract}

\section{Keywords: Communicating Converging and Diverging Channel, Numerical, Periodic Boundary Condition, Boundary Layer}

\section{INTRODUCTION}

It is very important to use compact heat exchangers in many engineering branches such as solar collectors, heaters, refrigerators, automotive and electronics equipment. Especially for the design of compact heat exchangers heat transfer must be maximized. Therefore, this area is investigated by many researchers [1-4].

There are many forms of heat transfer enhancements. Disrupting the growth of the boundary layer and mixing are one of the most applied methods for enhancement of heat transfer between the flowing fluid and heat transferring surfaces. For this application interrupted-wall channels were investigated, and it was showed that heat transfer can be increased using this method [5-8].

Converging and diverging channels are used in industry especially for increasing efficiency of heat exchangers. With these channels heat transfer surface area per volume is increased, boundary layers are disrupted and specially mixing regions are created to improve heat transfer coefficient. For this issue, there have been a lot of studies by researchers. Mendes and Sparrow [9] performed experimental study for the converging and diverging tubes to determine the entry and fully developed region heat transfer coefficients, pressure distribution and friction factor at various taper angles. Garg and Maji [10] numerically demonstrated that the converging and diverging channel configuration is an effective technique for enhancing of heat transfer.

Amon and Mikic [11] studied numerically heat transfer in interrupted channels and found the influence of non-steady state self-sustained oscillating flow. Herman et al. [12] showed experimentally the existence of oscillatory phenomena in a communicating channel.

Flow and heat transfer in periodic sinusoidal channels are investigated by Wang and Vanka [13] They showed that using these channels heat transfer can be enhanced with appropriate pressure drop.

Kotcioglu and Ayhan [14] accomplished evaluation of performance parameters in the turbulent regime regarding the enhancement of the heat transfer using winglet-type vortex generators. The heat transfer characteristics and flow structure in turbulent flow in a rectangular channel containing built-in wing type vortex generator, have been investigated experimentally. The vortices in this flow caused enhanced heat transfer. Dejond and Jacobi [15] investigated experimentally mass transfer at interrupted plate arrays and found that mass transfer is higher than theoretically calculated values for these systems. Converging and diverging communicating form of channel are used as finned surfaces by Caliskan and Baskaya [16] and Kotcioglu et al.[17]. Chunhua Min et al.[18] presented modified rectangular longitudinal vortex generator obtained by cutting off the four corners of a rectangular wing experimentally. Chunhua Min et al. [19] studied numerically comparative investigation longitudinal vortex generators for turbulent flow. Moreover, Gholami et al. [20] investigated winglet type vortex This paper was recommended for publication in revised form by Regional Editor Ahmet Selim Dalkılıç 
generators in fin and tube compact heat exchanger and found that vortex generators enhance thermal mixing of the fluid and delay the boundary layer separation on the tubes.

Heat transfer in communicating converging and diverging channels are first investigated theoretically and experimentally by Y1lmaz and Ayhan [21]. They have showed that due to mixture of the flow perpendicular to flow direction, heat transfer increases \%160 compared to parallel plate channels. They supplied experimental results which show that heat transfer enhancement increases with increasing Reynolds number.

There are lots of studies about implementation of periodic boundary condition in fluid flow and heat transfer analysis [22-24].

The communicating converging-diverging channels can be used in automobile radiators, PV collectors, gas-gas heat exchangers, liquid-liquid plate heat exchangers, etc. Numerical investigation of communicating converging diverging channel is introduced by Y1lmaz and Erdinç [25]. To the best of authors' knowledge there is not any other numerical investigation in the open literature concerning the communicating converging-diverging channels. In this study, detail numerical investigation is carried out on enhancement of forced convection heat transfer using communicating converging-diverging channels under the assumptions of two dimensional, viscous, incompressible, fully developed and laminar flow condition. For the validation of calculations, parallel plate channel is considered and obtained results were compared with the well-known values available in the literature for the convective heat transfer in channels

\section{MODEL DESCRIPTION}

Two-dimensional converging-diverging and communicating channel is shown in Figure 1. After a short entrance region, a periodic fully developed region is demonstrated in figure 2. It is assumed that the fluid is incompressible and laminar, constant wall temperature and steady-state conditions. The fluid properties are assumed to be constant.

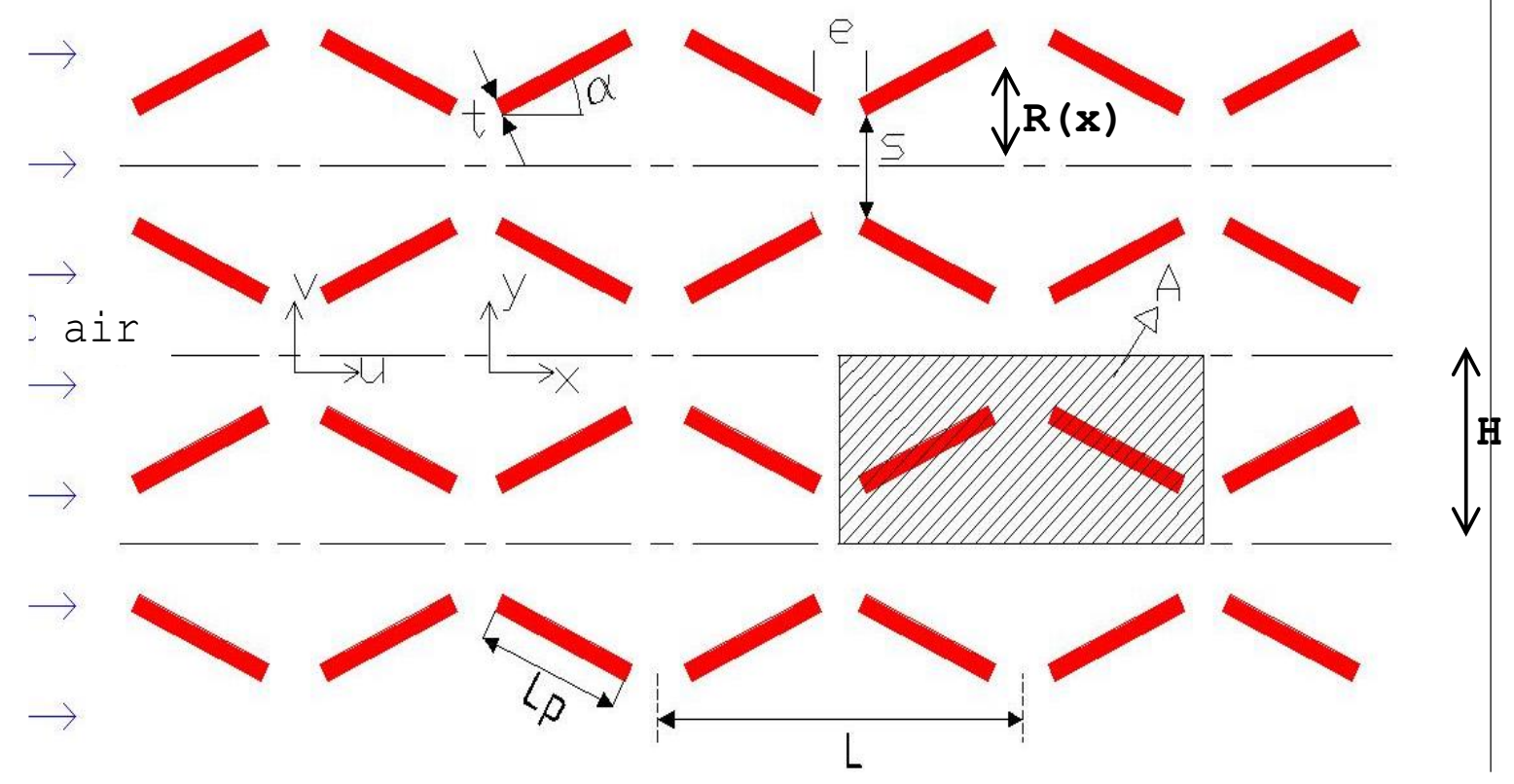

Figure 1. Converging-Diverging channel and computational domain A

For two-dimensional incompressible flow continuity and momentum equations for $\mathrm{x}$ and $\mathrm{y}$ direction can be written as follows [25]:

$$
\begin{gathered}
\frac{\partial u}{\partial x}+\frac{\partial v}{\partial y}=0 \\
u \frac{\partial u}{\partial x}+v \frac{\partial u}{\partial y}=-\frac{1}{\rho} \frac{\partial P}{\partial x}+\vartheta\left(\frac{\partial^{2} u}{\partial x^{2}}+\frac{\partial^{2} u}{\partial y^{2}}\right)
\end{gathered}
$$




$$
u \frac{\partial v}{\partial x}+v \frac{\partial v}{\partial y}=-\frac{1}{\rho} \frac{\partial P}{\partial y}+\vartheta\left(\frac{\partial^{2} v}{\partial x^{2}}+\frac{\partial^{2} v}{\partial y^{2}}\right)
$$

For two dimensional, steady, incompressible and laminar flow energy equation is written as:

$$
u \frac{\partial T}{\partial x}+v \frac{\partial T}{\partial y}=a\left(\frac{\partial^{2} T}{\partial x^{2}}+\frac{\partial^{2} T}{\partial y^{2}}\right)
$$

Here $a$ is thermal diffusity.

The associated boundary conditions are demonstrated for hydrodynamically fully developed flow and are presented in figure 2 and these are given below:

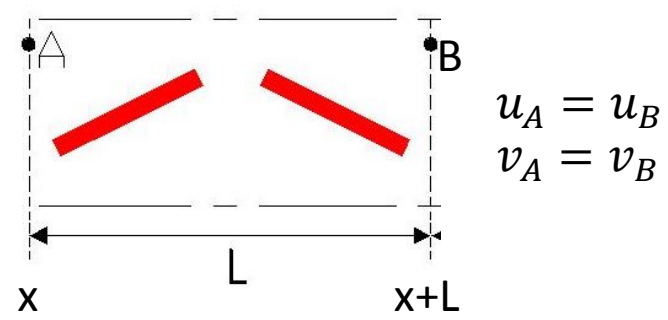

Figure 2. Presentation of developed flow boundary condition for in converging-diverging and communicating channel

$$
\begin{gathered}
u(y, x)=u(y, x+L) \\
v(y, x)=v(y, x+L) \\
p(y, x)=p(y, x+L)+\Delta p_{p}
\end{gathered}
$$

where $\Delta P_{p}$ is pressure loss in one periodic element of the length $\mathrm{L}$ in the channel.

For the temperatures at $\mathrm{x}$ and $\mathrm{x}+\mathrm{L}$, we can write

$$
\frac{T(y, x)-T_{\text {wall }}}{\bar{T}(x)-T_{\text {wall }}}=\frac{T(y, x+L)-T_{\text {wall }}}{\bar{T}(x+L)-T_{\text {wall }}}
$$

where $\bar{T}(x)$ and $\bar{T}(x+L)$ are mean temperatures at $\mathrm{x}$ and $\mathrm{x}+\mathrm{L}$. Mean temperature is defined as follows:

$$
\bar{T}(x)=\frac{\int_{0}^{R(x)} u T y d y}{\int_{0}^{R(x)} u y d y}
$$

$R(x)$ is shown in figure 1. For symmetry boundary conditions below equation can be written:

$$
\frac{\partial u(x, y)}{\partial y}=0, \quad v(x, y)=0, \quad \frac{\partial T(x, y)}{\partial y}=0
$$

Reynolds number and equivalent diameter are defined as follows: 


$$
\begin{gathered}
R e=\frac{\rho u_{m} d_{e}}{\mu} \\
d_{e}=\frac{4 \cdot V}{F_{p}}=\frac{(H \cdot L)}{\left(L_{p}+t\right)}
\end{gathered}
$$

where $\mathrm{H}$ is the width of the channel and $u_{m}$ is mean velocity of air at the inlet of the computational domain.

\section{SOLUTION}

The steady, laminar and incompressible Navier-Stokes equations were discretized using the finite volume method for flow domain demonstrated in figure 1 and 3. ANSYS FLUENT computer program was used to solve the governing equations. QUICK scheme was applied for convective and diffusive terms. The pressure-velocity coupling algorithm SIMPLE was selected. Meshes were implemented to the flow domain as illustrated in figure 4. The check of mesh independence is necessarily to ensure the accuracy and validity of the numerical results. So, the mesh independence study is conducted on the all channels. The four parameters of interest for the present case are friction factor, Nusselt number, mixing ratio and thermal enhancement factor. Convergence criteria for momentum and continuity equations are $10^{-6}$ and convergence criteria for energy equations are $10^{-9}$.

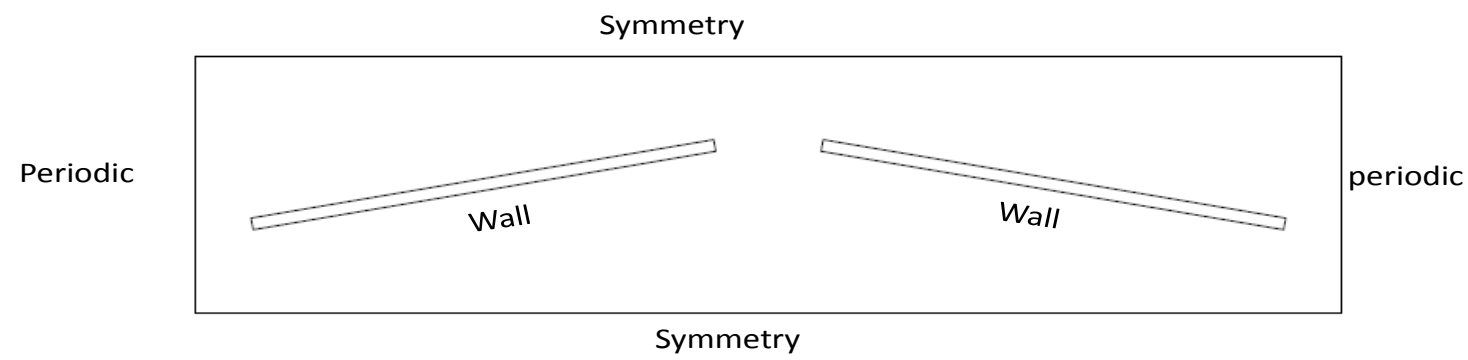

Figure 3. Boundary conditions of flow domain

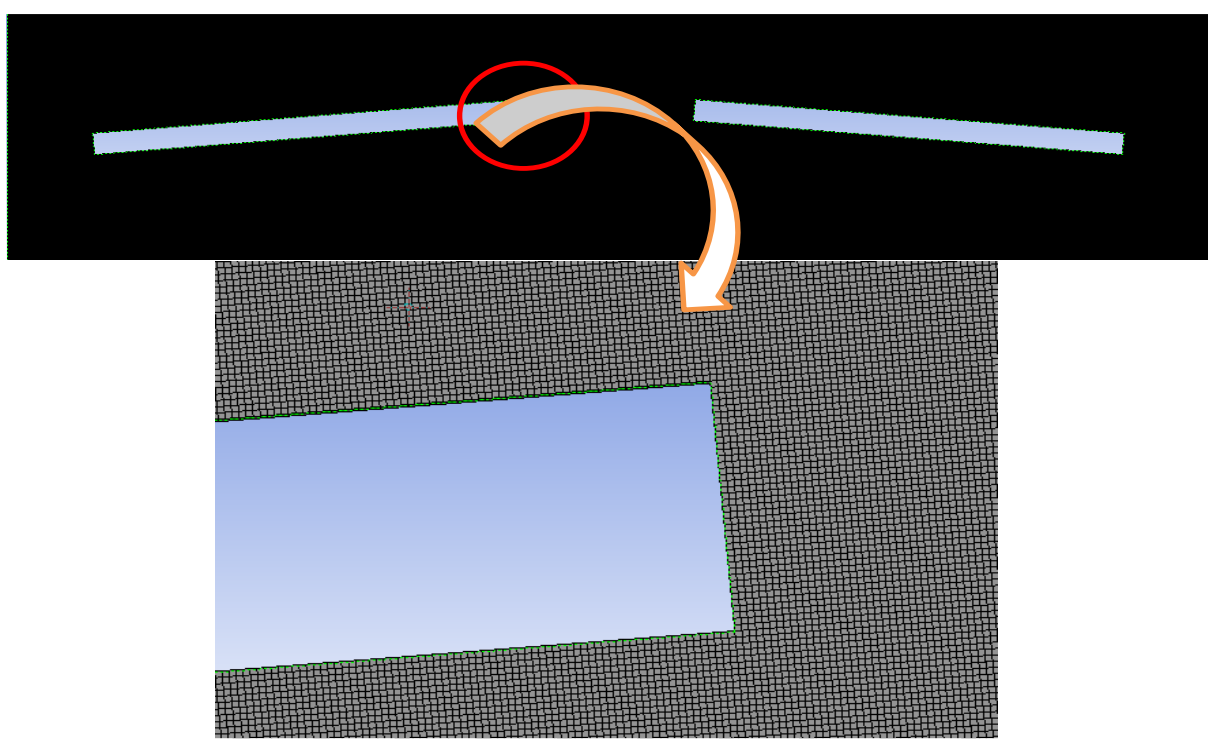

Figure 4. Presentation of mesh

Local Nusselt number is calculated according to the equation below:

$$
N u_{x}=\frac{h(x) d_{e}}{k}
$$

where $\mathrm{h}(\mathrm{x})$ and $\mathrm{k}$ are local heat transfer coefficient and thermal conductivity of air respectively. $\mathrm{h}(\mathrm{x})$ is defined as follows: 


$$
h(x)=\frac{\dot{q}_{x}}{T_{w}-\bar{T}(x)}
$$

$\dot{q}_{x}, \bar{T}(x)$ are heat flux at the point $\mathrm{x}$ and mean temperature.

Mean heat transfer coefficient and Nusselt number are determined as follows:

$$
h=\frac{\dot{Q}}{F_{p} \Delta T_{m}}
$$

where $F_{p}$ is plate surface area:

$$
F_{p}=4 \cdot\left(L_{p}+t\right) \cdot B
$$

and $\mathrm{B}$ is the width of the channel.

$\dot{Q}$ is determined from the equation

$$
\dot{Q}=\dot{M} \cdot c_{p} \cdot\left(T_{e}-T_{i}\right)=h \cdot F_{p} \cdot \Delta T_{m}
$$

where $\dot{M}, c_{p}, T_{i}, T_{e}$ are mass flow rate, specific heat and fluid inlet/exit temperature at the inlet and exit of the computational domain. $\Delta T_{m}$ is logarithmic mean temperature difference:

$$
\Delta T_{m}=\frac{\left(T_{w}-T_{i}\right)-\left(T_{w}-T_{e}\right)}{\ln \frac{\left(T_{w}-T_{i}\right)}{\left(T_{w}-T_{e}\right)}}
$$

Mean Nusselt numbers are calculated then as:

$$
N u=\frac{h d_{e}}{k}
$$

Friction factor is defined as usual:

$$
f=\frac{\Delta P_{p} d_{e} / L}{\rho \frac{u_{m}^{2}}{2}}
$$

where $\rho$ is density of the fluid.

Thermal enhancement factor is defined as follows [26]:

$$
\eta=\frac{\frac{N u}{N u_{p}}}{\left(\frac{f}{f_{p}}\right)^{1 / 3}}
$$

$\mathrm{Nu}, f$ and $\mathrm{Nu}_{\mathrm{p}}, \mathrm{f}_{\mathrm{p}}$ are Nusselt number and friction factors for the calculated channels and parallel plate channels respectively.

For the numerical solution temperature and geometric parameters are given in table 1 and 2. 
Table 1. Boundary conditions

\begin{tabular}{|l|c|}
\hline Boundary & Value \\
\hline $\boldsymbol{T}_{\boldsymbol{w}}$ (Wall) & $363.15 \mathrm{~K}$ \\
\hline $\boldsymbol{T}_{\boldsymbol{a i r}}$ (inlet) & $303.15 \mathrm{~K}$ \\
\hline
\end{tabular}

Table 2. Geometric dimensions of flow domain.

\begin{tabular}{|l|c|}
\hline Geometric parameter & Dimension \\
\hline $\mathrm{L}$ & $40 \mathrm{~mm}$ \\
\hline $\mathrm{t}$ & $2 \mathrm{~mm}$ \\
\hline $\mathrm{e}$ & $10 \mathrm{~mm}$ \\
\hline $\mathrm{H}$ & $25 \mathrm{~mm}$ \\
\hline$\alpha$ & $0-30^{\circ}$ \\
\hline
\end{tabular}

\section{RESULTS AND DISCUSSIONS}

Since there is not any study to compare with this system, validation of the heat transfer and friction factor of the parallel plate is performed by comparing with the previous values under similar operating conditions for hydrodynamically and thermally fully developed flow.

As seen in figure 5 and 6 , the present numerical results are found to be in good agreement with exact solution values obtained from the literature [27] for both the Nusselt number and the friction factor. Also flow in interrupted wall channel without angle is compared with Yilmaz [5] using same parameters. Yilmaz [5]'s results are shown in figure 7. Numerical results are again found to be in good agreement with the results obtained by Yilmaz [5] as seen in figure 8 for $L^{*}=40$. This shows a strong confidence in further investigation of the communicating converging diverging channels.

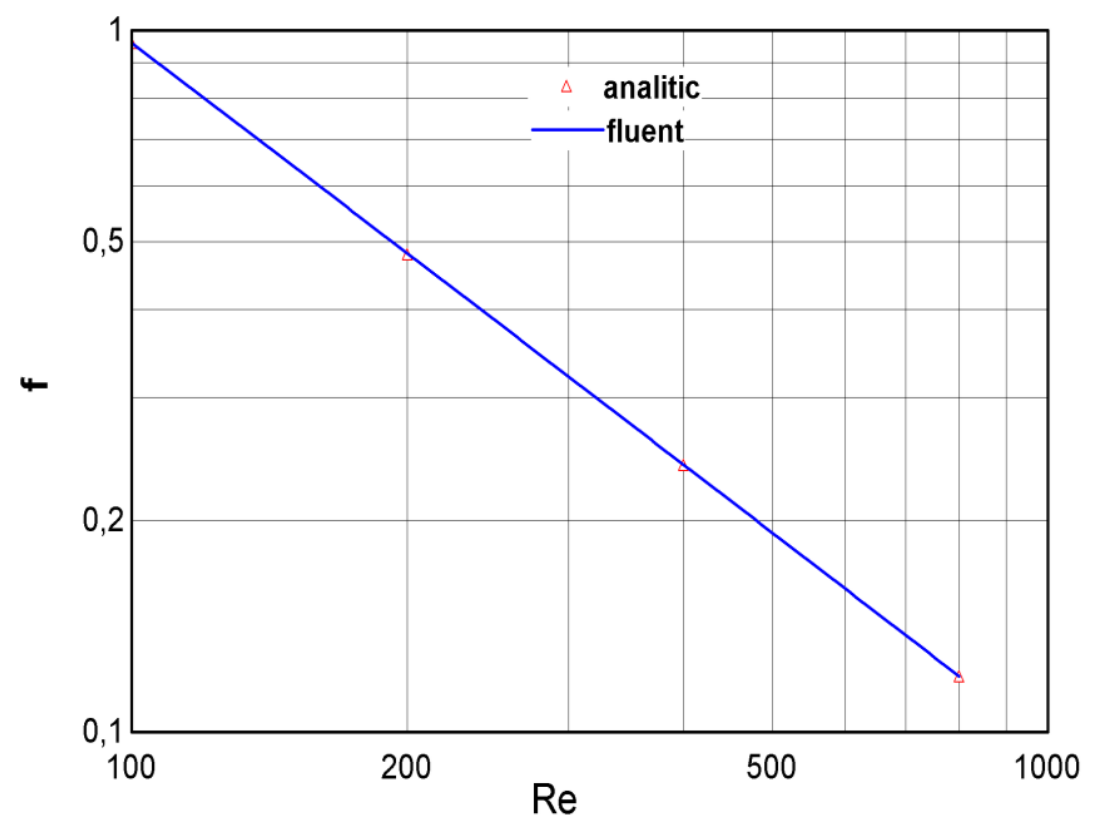

Figure 5. Comparison of friction factor between the values calculated in this work and analytical results for parallel plate channel. 
Journal of Thermal Engineering, Research Article, Vol. 4, No. 5, pp. 2318-2332, July, 2018

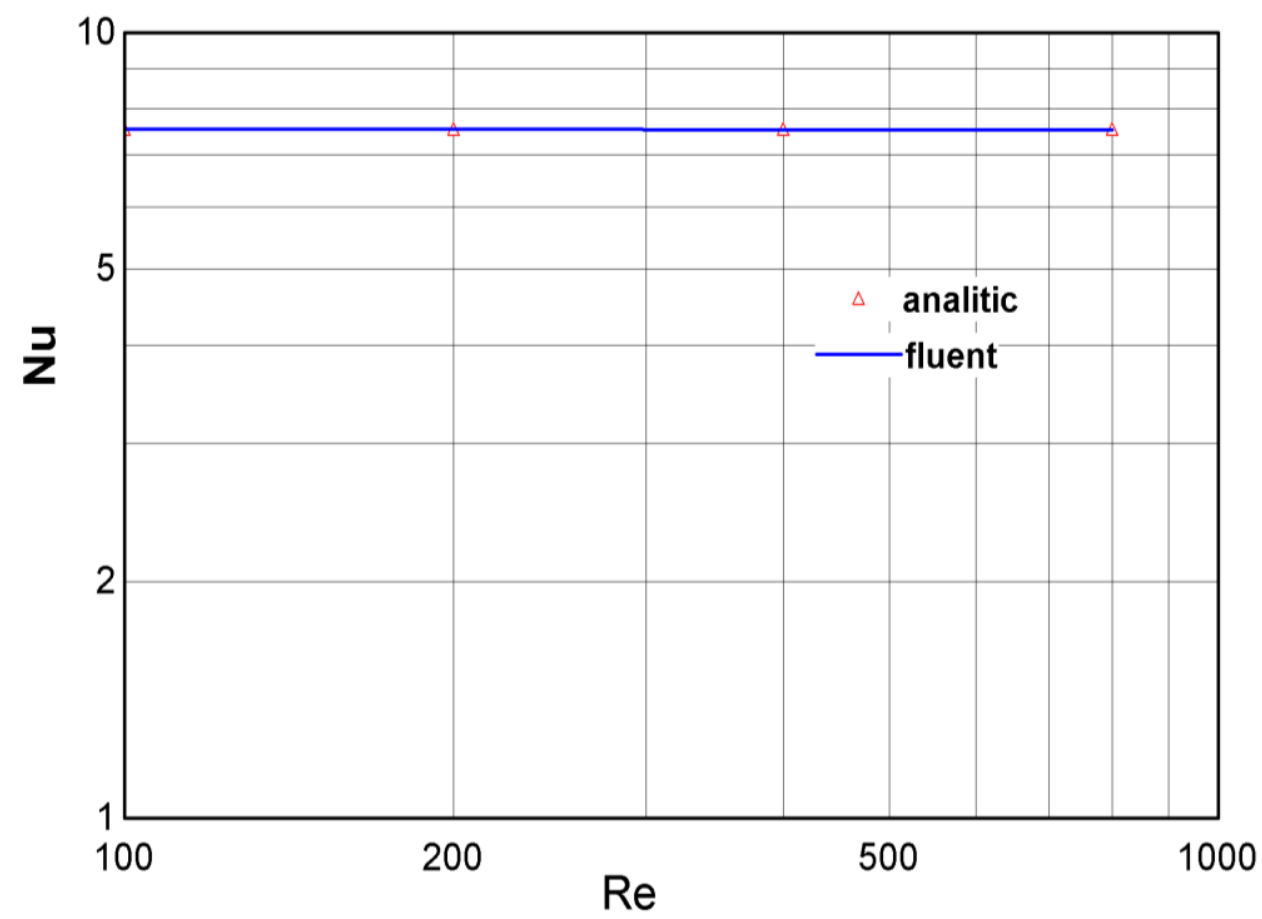

Figure 6. Comparison of Nusselt number between the values calculated in this work and analytical results for parallel plate channel.

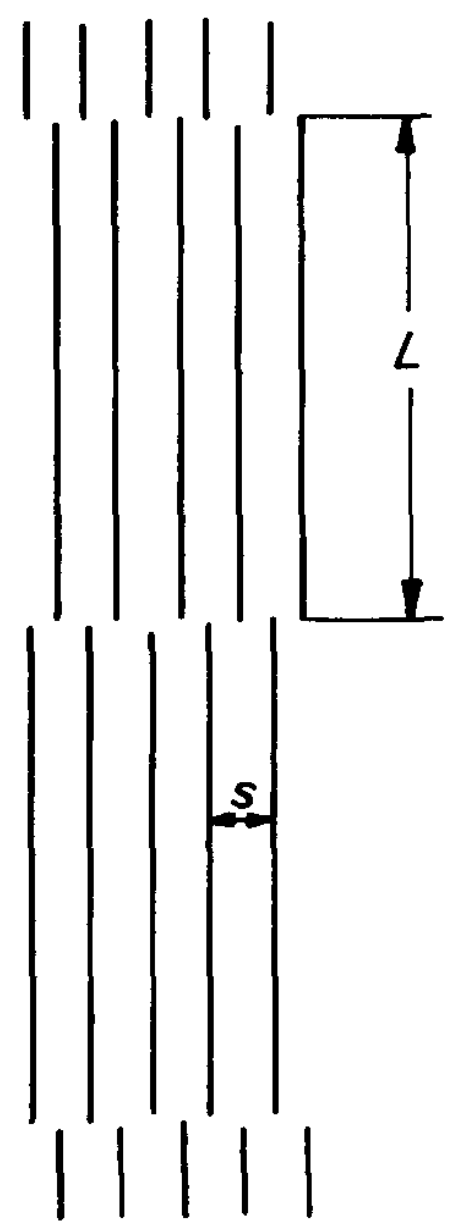

Figure 7. Arrangement of plates in Yilmaz [5]'s work. 


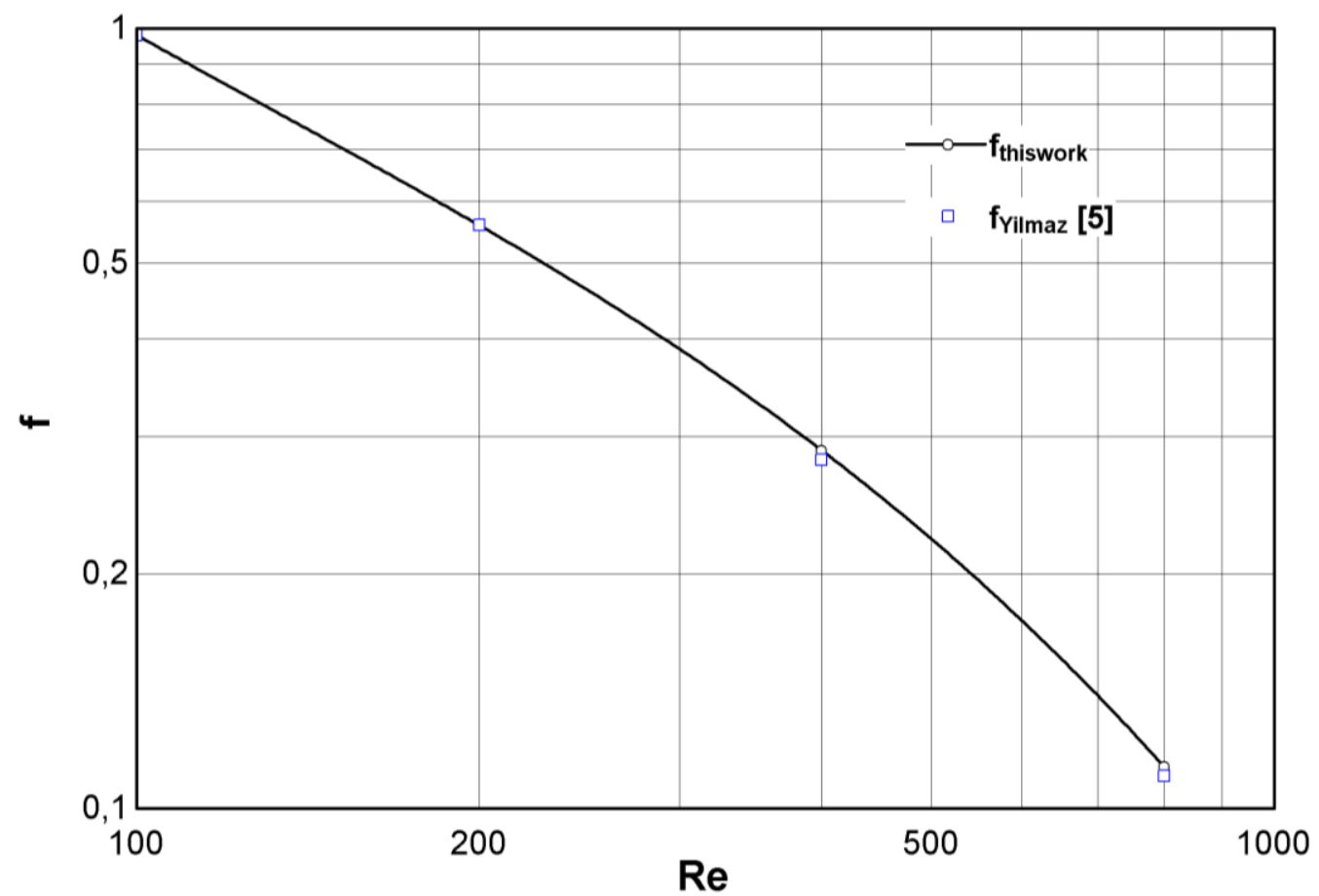

Figure 8. Comparison of friction factor between the values calculated in this work and Yilmaz [5]'s work for $L^{*}=40\left(L^{*}=L / 2 s\right)$

It is expected that there exists a certain optimum angle for these channels. Therefore, especially this optimum value of the angle should be determined. For a given Reynolds number $(\mathrm{Re}=100)$, the mixing effect of the angle $(\alpha)$ is investigated. Flow planes for the explanation of the flow are shown in figure 9.

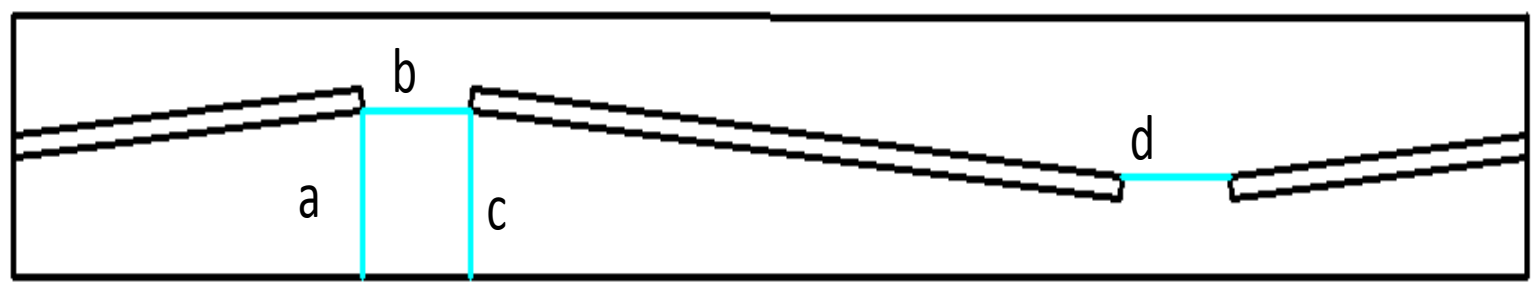

Figure 9. Flow areas

To show the mixing effect of the flow between the channels a mixing ratio is defined:

$$
\dot{M}^{*}=\frac{\dot{M}_{b}}{\dot{M}_{a}}
$$

$\dot{\mathrm{M}}_{\mathrm{b}}$ and $\dot{\mathrm{M}}_{a}$ are mass flow rate of fluid passing through area $\mathrm{b}$ and $\mathrm{a}$. Calculated mixing ratios are given in table 3 and shown in figure 10 . As seen from the figure 10, there is a maximum mixing point at $\alpha=20^{\circ}$. At the maximum point, $26 \%$ of the mass flow is transferred to the neighboring channel.

Figures $11,12,13$ and 14 respectively show velocity distributions and streamlines for $\mathrm{Re}=100$. The figures indicate that communicating converging and diverging channels not only can interrupt thermal boundary layer, but also enhance mixing of the fluid. This is due to pressure and velocity differences across the passages between converging-diverging channels. For different angles $\alpha$, mixing of the flow in adjacent channels are clearly seen in these figures. However, vortex formation occurs after certain plate angle of $15^{\circ}$, and this causes rapid increase of pressure drop. 
Table 3. Effect of angle $(\alpha)$ on mixing ratio

\begin{tabular}{|c|c|}
\hline $\boldsymbol{\alpha}$ & $\dot{\mathbf{M}}^{*}$ \\
\hline 5 & 0.1055 \\
\hline 10 & 0.1916 \\
\hline 15 & 0.2479 \\
\hline 20 & 0.2664 \\
\hline 25 & 0.2569 \\
\hline 30 & 0.2382 \\
\hline
\end{tabular}

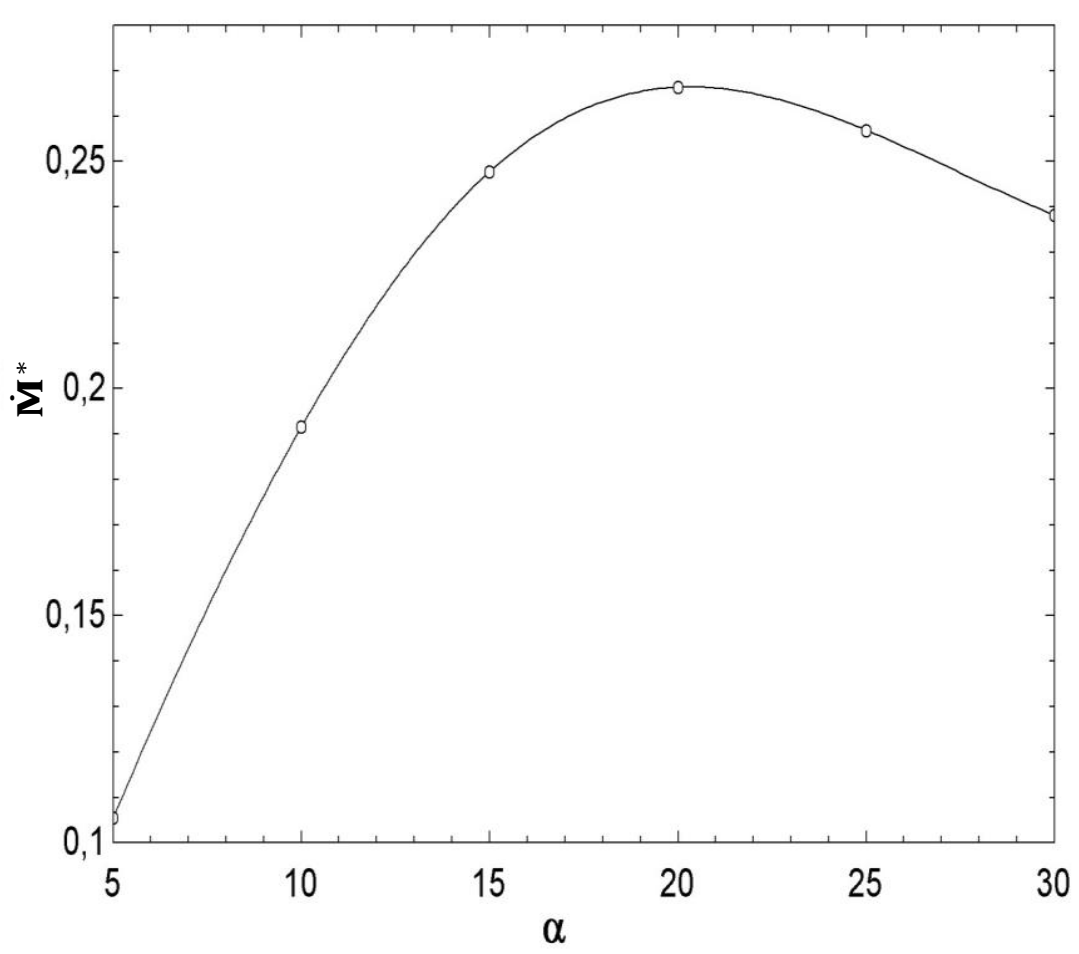

Figure 10. Effect of angle $(\alpha)$ on mixing ratio

Figure 17 shows the thermal enhancement factor for such channels for different plate angle in the Re range of 100-600. As outlined above, due to vortex formation thermal enhancement factor is greater than 1 for $\alpha \leq 15$. For $\alpha \geq 15$, as Reynolds number increases, thermal enhancement factor increase also. The thermal performance factor above unity indicated that the effect of heat transfer enhancement due to the enhancing device was more dominant than the effect of rising friction and vice versa [28].

Effect of plate angle on friction factor and Nusselt number for different Reynold numbers are shown in figure 15 and 16. As plate angle increases Nusselt number increases because of mixing effect and vortex formation. However, friction factor increases due to intensive vortex formation with the increase of plate angle. It can be seen also that when Re number increases; Nusselt number increases, and friction factor decreases as expected. 
Journal of Thermal Engineering, Research Article, Vol. 4, No. 5, pp. 2318-2332, July, 2018

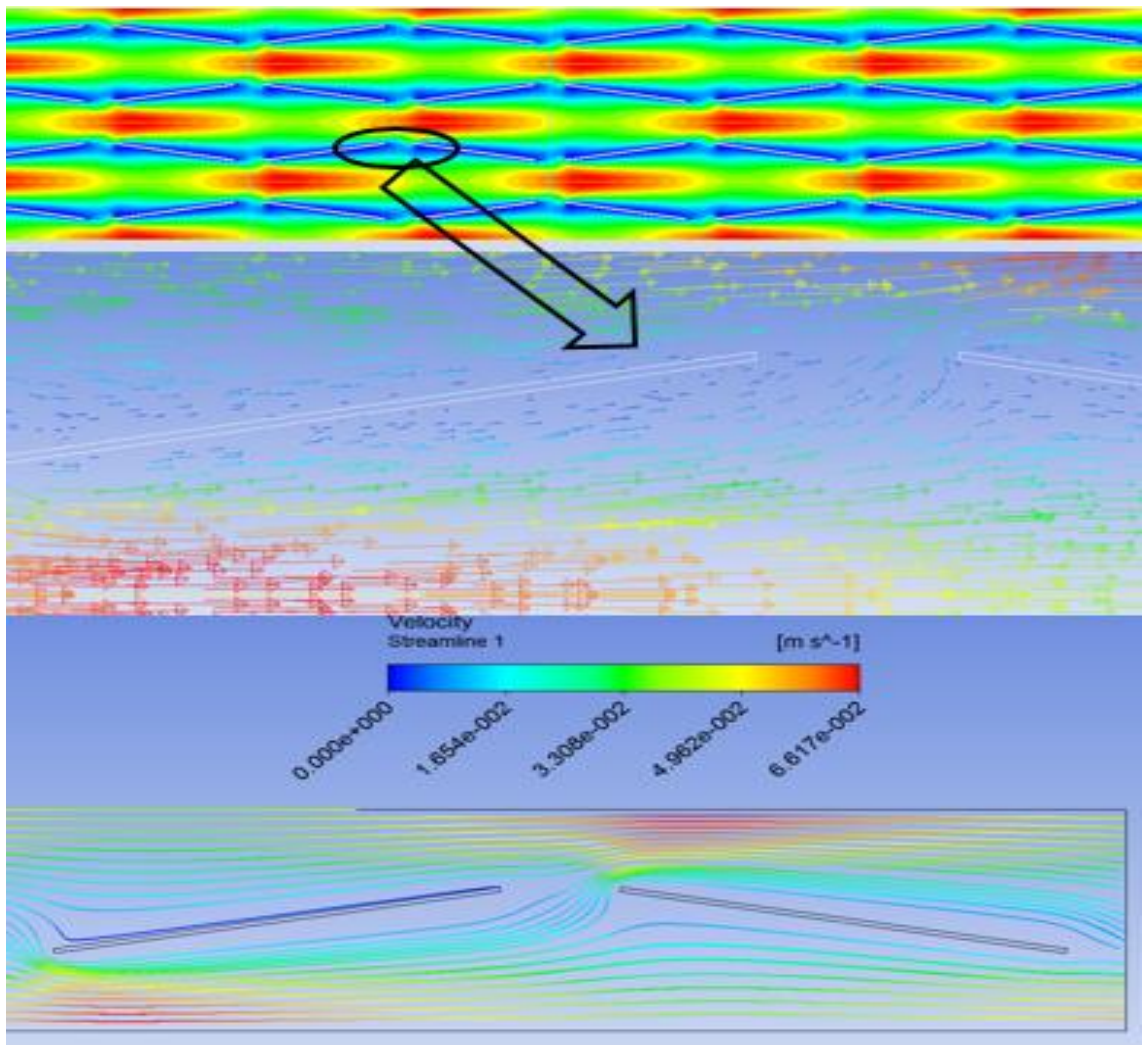

Figure 11. Velocity vectors and streamlines for $\alpha=\mathbf{1 0}^{\circ}$

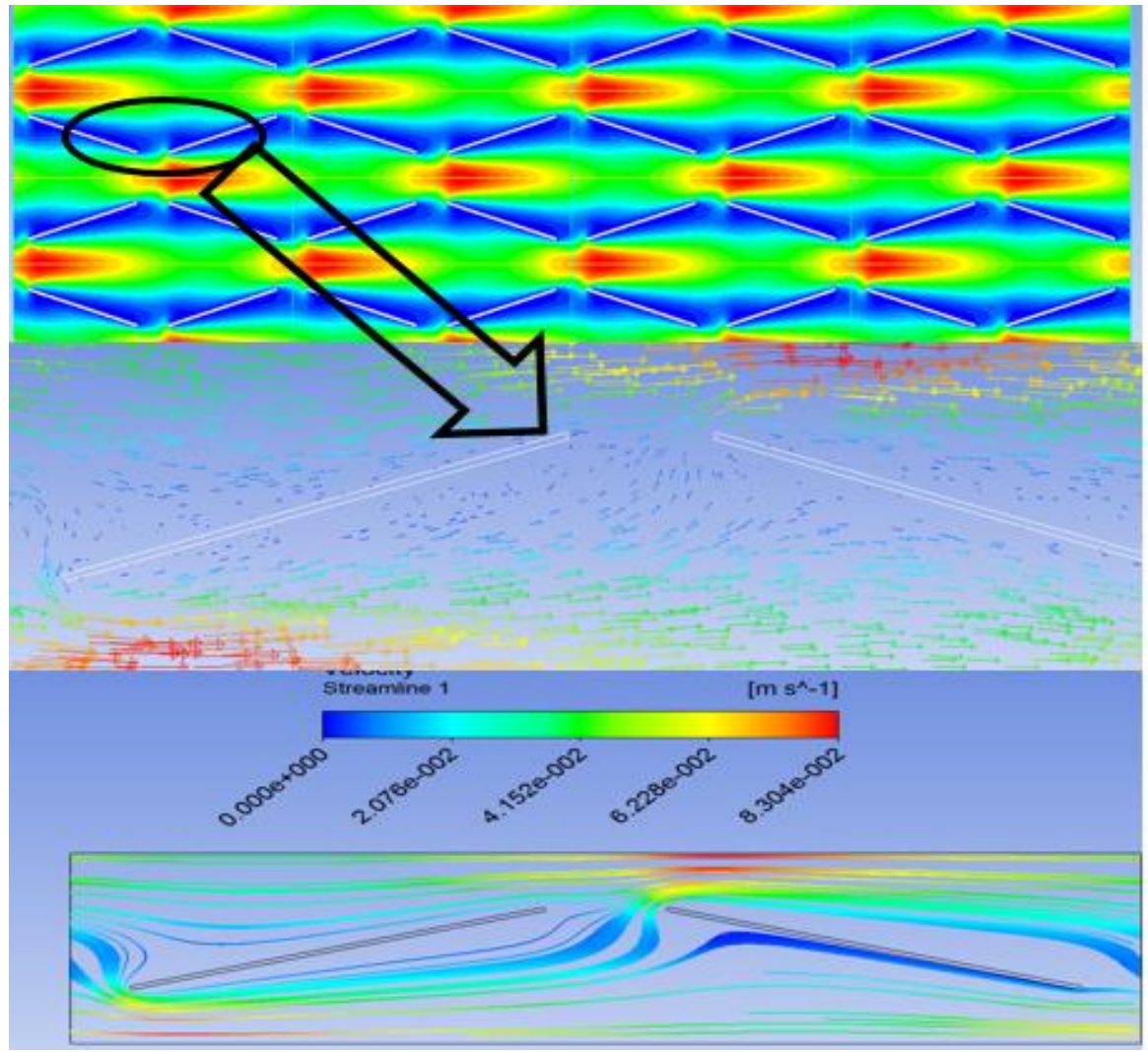

Figure 12. Velocity vectors and streamlines for $\boldsymbol{\alpha}=\mathbf{1 5}^{\circ}$ 
Journal of Thermal Engineering, Research Article, Vol. 4, No. 5, pp. 2318-2332, July, 2018

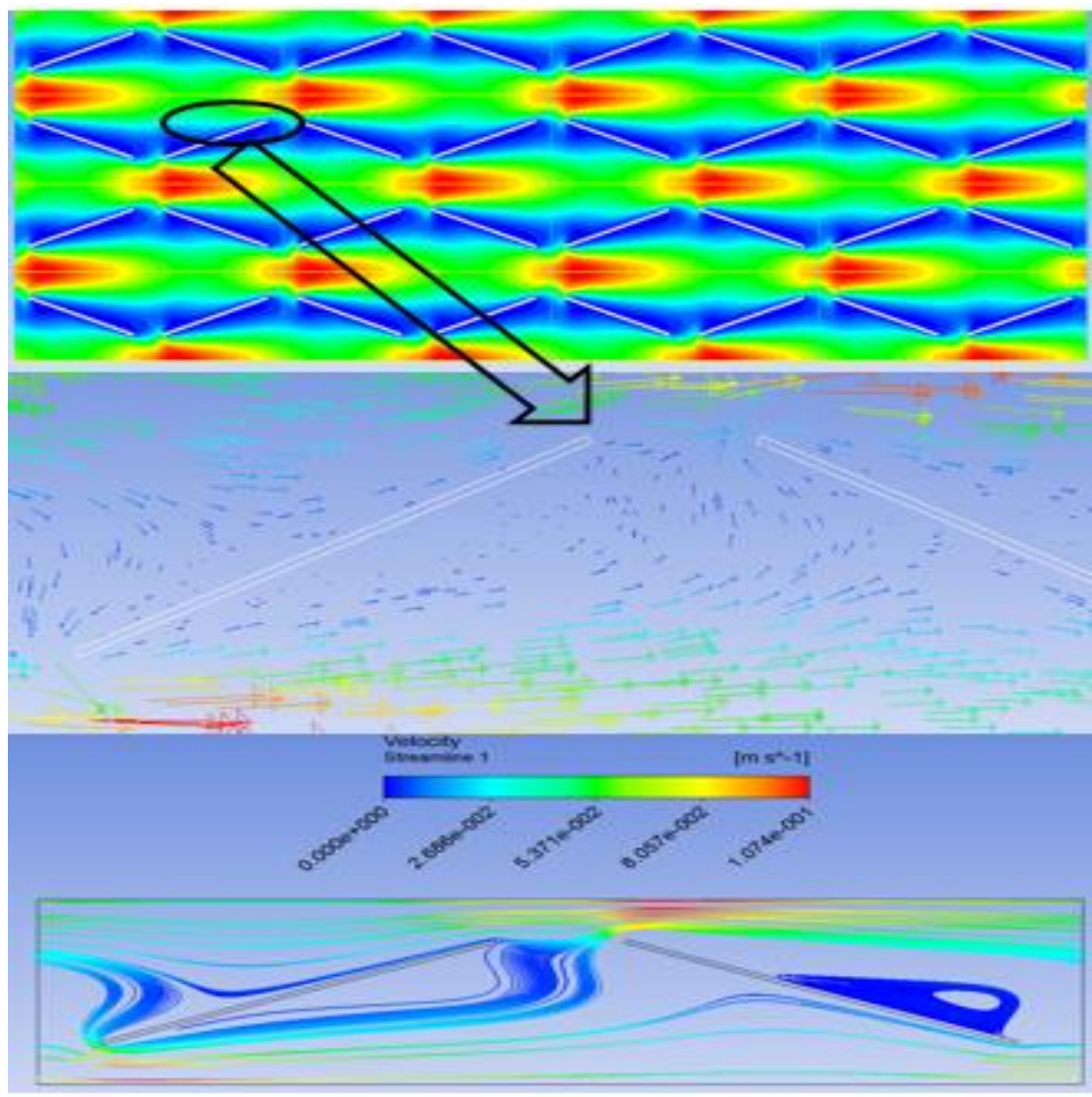

Figure 13. Velocity vectors and streamlines for $\boldsymbol{\alpha}=\mathbf{2 0}^{\circ}$

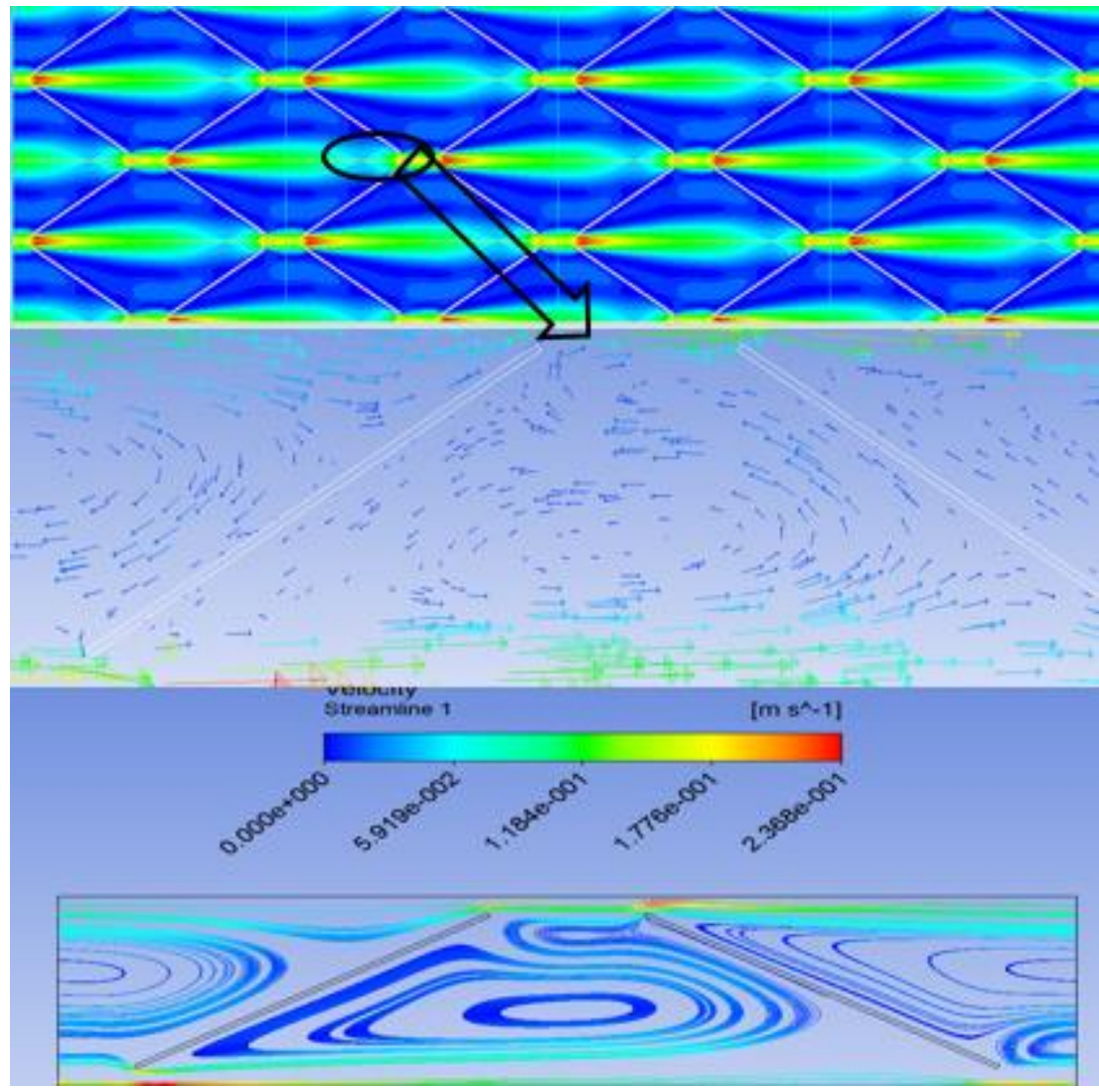

Figure 14. Velocity vectors for $\boldsymbol{\alpha}=\mathbf{3 0}^{\circ}$ 
Journal of Thermal Engineering, Research Article, Vol. 4, No. 5, pp. 2318-2332, July, 2018

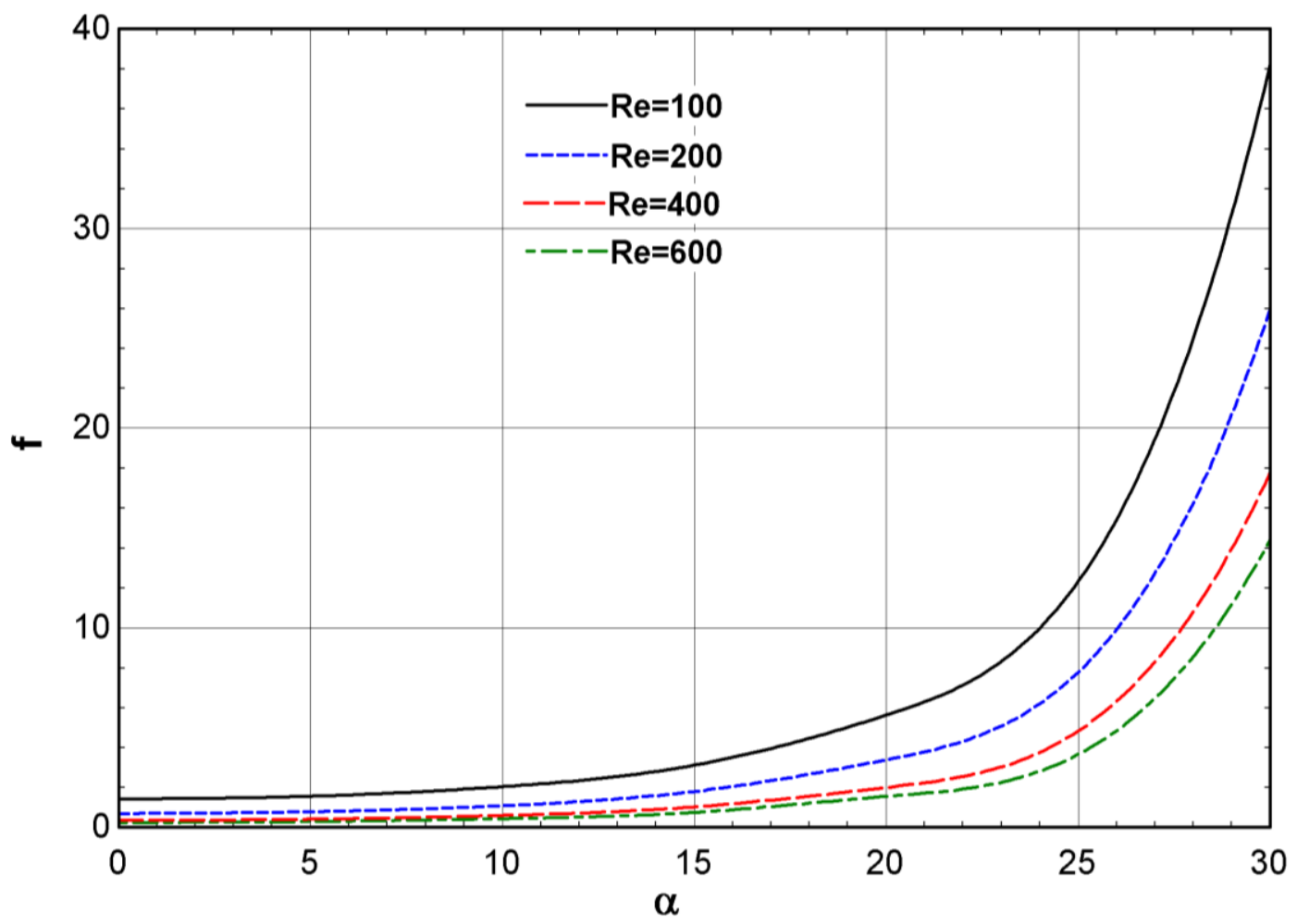

Figure 15. Variation of friction factor with plate angle for different Reynolds number

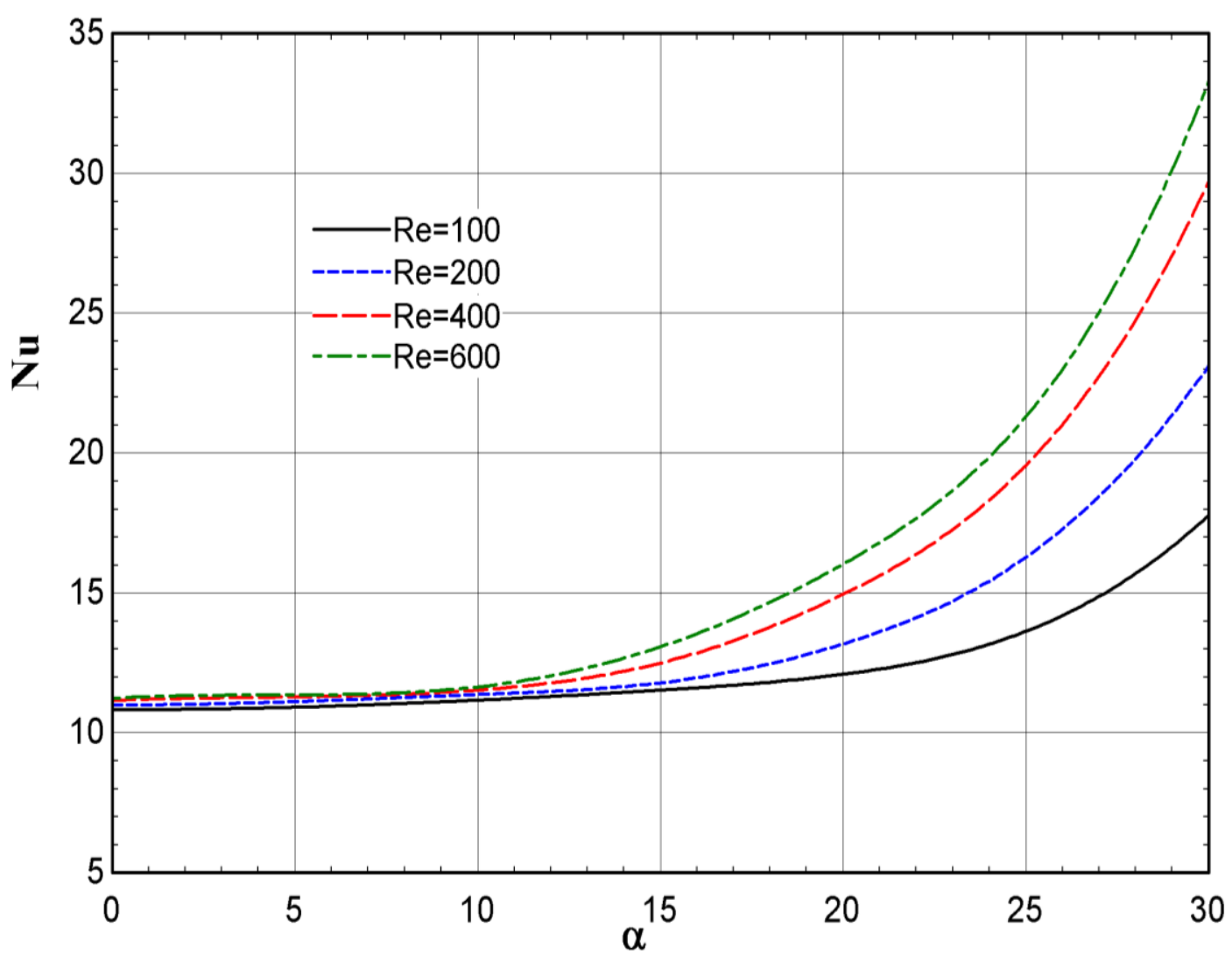

Figure 16. Variation of Nusselt number with plate angle for different Reynolds number 


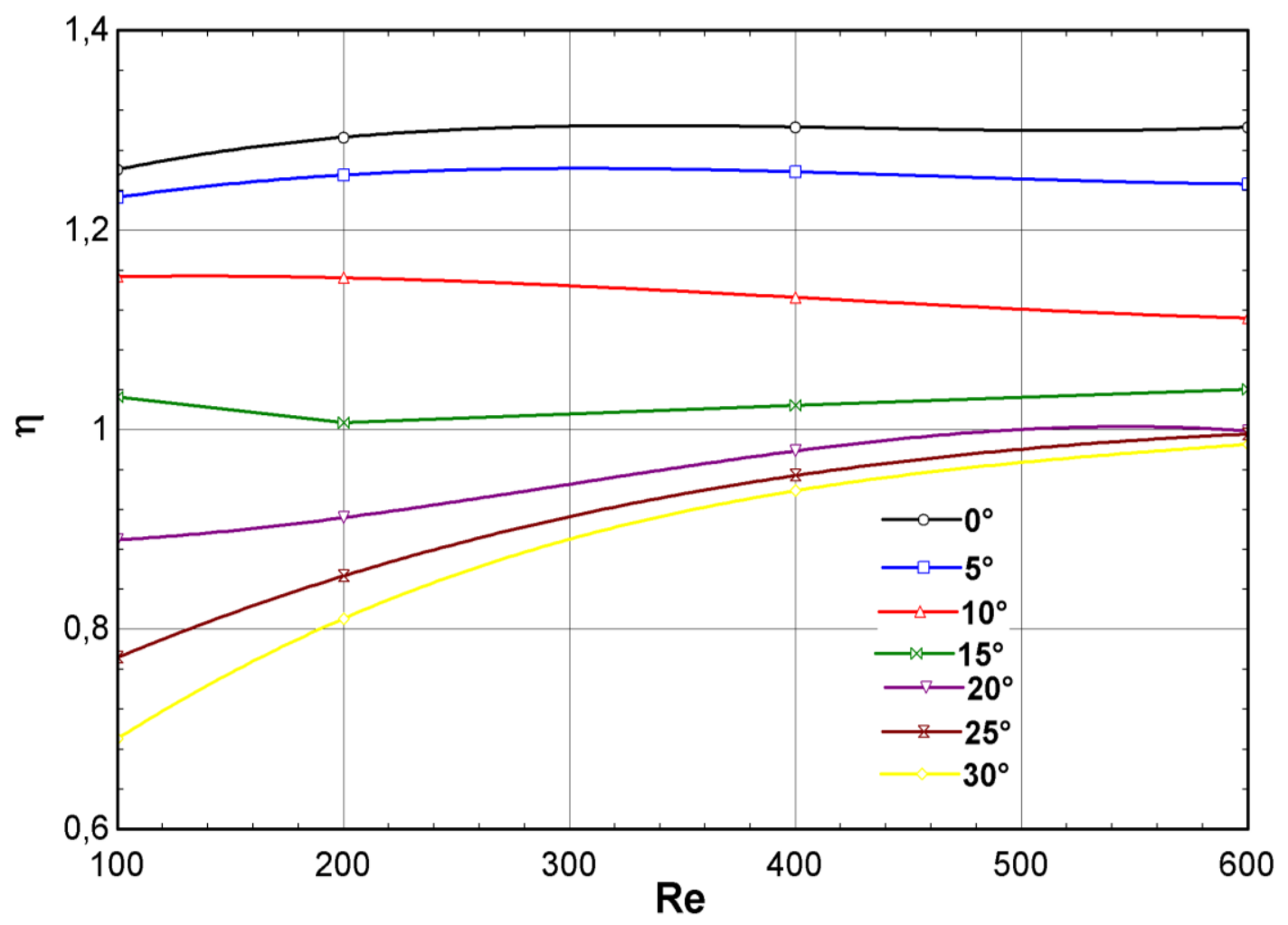

Figure 17. Variation of thermal enhancement factor with Reynolds number for different plate angle

\section{CONCLUDING REMARKS}

Two-dimensional numerical investigation of flow and heat transfer in communicating convergingdiverging channel has been performed under assumptions of viscous, incompressible fully developed and laminar flow condition. Especially mixing of the fluid in adjacent channels are determined and illustrated. The following conclusions can be drawn:

- Due to pressure and velocity differences across the passages between converging-diverging channels, mixing effect between channels is obtained.

- The converging-diverging channels mix the fluid perpendicular to the flow directions and destroy the boundary layer significantly and thereby increase the heat transfer coefficient to develop a more effective heat exchanger.

- It is shown that at $\mathrm{Re}=100$ maximum mixing occurs at the angle $\alpha=20^{\circ}$.

- Nusselt number and friction factor are increasing with the increasing of angle $\alpha$ and Reynolds number

- Thermal enhancement factor is higher than 1 for $\alpha<15^{\circ}$.

- These channels can be used in automobile radiators, PV collectors, gas-gas heat exchangers, liquid-liquid plate heat exchangers and etc.

\section{ACKNOWLEDGEMENTS}

This study is supported by Osmaniye Korkut Ata University Scientific Research Project Department with Project No. OKÜBAP 2013- PT3-022.

\section{NOMENCLATURE}

$c_{p} \quad$ specific heat $[\mathrm{J} / \mathrm{kg}]$

$d_{e} \quad$ equivalent diameter[m]

f friction factor, eq.(20)

$F_{p} \quad$ heat transfer surface area $\left[\mathrm{m}^{2}\right]$

$\mathrm{h} \quad$ heat transfer coefficient $\left[\mathrm{W} / \mathrm{m}^{2} . \mathrm{K}\right]$ 
$\mathrm{H}$ height of the computational area[m]

$\mathrm{k}$ thermal conductivity of the fluid [W/m.K]

$\mathrm{L} \quad$ length of computational domain[m]

$\mathrm{L}_{\mathrm{p}} \quad$ length of plate[m]

$\dot{M} \quad$ mass flow rate of the fluid $[\mathrm{kg} / \mathrm{s}]$

$\mathrm{Nu} \quad$ Nusselt number, eqs.(13), (19)

$\mathrm{s} \quad$ minimum channel distance $[\mathrm{m}]$

$\dot{q} \quad$ heat flux $\left[\mathrm{W} / \mathrm{m}^{2}\right]$

$\dot{Q} \quad$ heat flow[W]

$\mathrm{t}$ thickness of plate[m]

$\mathrm{T} \quad$ temperature[K]

$\mathrm{u} \quad$ velocity $[\mathrm{m} / \mathrm{s}]$

$\mathrm{V} \quad$ volume$\left[\mathrm{m}^{3}\right]$

$\mathrm{R} \quad$ distance between axis and wall[ $[\mathrm{m}]$

$\mathrm{y} \quad$ coordinate perpendicular to $\mathrm{x}[\mathrm{m}]$

$\mathrm{x} \quad$ axial coordinate $[\mathrm{m}]$

$\alpha \quad$ angle of the plate

$\rho \quad$ density of the fluid $\left[\mathrm{kg} / \mathrm{m}^{3}\right]$

$\Delta P \quad$ pressure loss $[\mathrm{Pa}]$

$\Delta T_{m} \quad$ logarithmic mean temperature difference[K]

b bulk

e equivalent, exit

i inlet

$\mathrm{p} \quad$ plate

w wall

$\mathrm{x}$ local

\section{REFERENCES}

[1] Jasim, H. H., Söylemez, M. S. (2016). Enhancement of Natural Convection Heat Transfer of Pin Fin Having Perforated With Inclination Angle. Isi Bilimi ve Teknigi Dergisi/Journal of Thermal Science \& Technology, 36(2). [2] Jasjeevan, S., Ranjit, S., Brij B. (2015). Thermo hydraulic performance of solar air duct having triangular protrusions as roughness geometry, Journal of Thermal Engineering, 1:2, Special Issue 7, 607-620.

[3] Tokgöz, N., Aksoy, M. M., Şahin, B. (2016). Experimental investigation of flow characteristics of corrugated channel flow using PIV, Journal of Thermal Engineering, 2 (2), 754-760.

[4] Zunaid, M. Jindal, A., Gakhar, D., Sinha, A. (2017). Numerical study of pressure drop and heat transfer in a straight rectangular and semi cylindrical projections microchannel heat sink, Journal of Thermal Engineering, 3(5),1453-1465.

[5] Yilmaz, T. Flow (1977). heat and mass transfer in staggered plate rows (Turkish), Associate Professor Thesis. K.T. Ü.

[6] Sparrow, E. M., Baliga, B. R., Patankar, S. V. (1977). Heat transfer and fluid flow analysis of interrupted-wall channels, with application to heat exchangers. Journal of Heat Transfer, 99(1), 4-11.

[7] Patankar, S. V., \& Prakash, C. (1981). An analysis of the effect of plate thickness on laminar flow and heat transfer in interrupted-plate passages. International Journal of Heat and Mass Transfer, 24(11), 1801-1810.

[8] Yilmaz, T. (1982). Numerical solution of Navier-stokes equations for laminar fluid flow in rows of plates in staggered arrangement. International Journal of Heat and Fluid Flow, 3(4), 201-206.

[9] Mendes, P. S., Sparrow, E. M. (1984). Periodically converging-diverging tubes and their turbulent heat transfer, pressure drop, fluid flow, and enhancement characteristics. Journal of heat transfer, 106(1), 55-63.

[10] Garg, V. K., Maji, P. K. (1988). Laminar flow and heat transfer in a periodically converging-diverging channel. International journal for numerical methods in fluids, 8(5), 579-597. 
[11] Amon, C. H., Mikic, B. B. (1990). Numerical prediction of convective heat transfer in self-sustained oscillatory flows. Journal of thermophysics and heat transfer, 4(2), 239-246.

[12] Herman, C. V., Mayinger, F., Sekulic, D. P. (1991). Experimental verification of oscillatory phenomena in heat transfer in a communicating channels geometry. In Proc. of the 2nd World Conf. on Exp. Heat Transfer, Fluid Mechanics and Thermodynamics, Dubrovnik, Yugoslavia (904-911).

[13] Wang, G. V., Vanka, S. P. (1995). Convective heat transfer in periodic wavy passages. International Journal of Heat and Mass Transfer, 38(17), 3219-3230.

[14] Kotcioğlu, İ., Ayhan, T., Olgun, H., Ayhan, B. (1998). Heat transfer and flow structure in a rectangular channel with wing-type vortex generator. Turkish Journal of Engineering and Environmental Sciences, 22(3), 185-196.

[15] DeJong, N. C., Jacobi, A. M. (1997). An experimental study of flow and heat transfer in parallel-plate arrays: local, row-by-row and surface average behavior. International Journal of Heat and Mass Transfer, 40(6), 1365 1378.

[16] Caliskan, S., Baskaya, S. (2012). Experimental investigation of impinging jet array heat transfer from a surface with V-shaped and convergent-divergent ribs. International Journal of Thermal Sciences, 59, 234-246.

[17] Kotcioglu, I., Cansiz, A., Khalaji, M. N. (2013). Experimental investigation for optimization of design parameters in a rectangular duct with plate-fins heat exchanger by Taguchi method. Applied Thermal Engineering, 50(1), 604-613.

[18] Min, C., Qi, C., Kong, X., Dong, J. (2010). Experimental study of rectangular channel with modified rectangular longitudinal vortex generators. International Journal of Heat and Mass Transfer, 53(15-16), 3023-3029. [19] Min, C., Qi, C., Wang, E., Tian, L., Qin, Y. (2012). Numerical investigation of turbulent flow and heat transfer in a channel with novel longitudinal vortex generators. International Journal of Heat and Mass Transfer, 55(2324), 7268-7277.

[20] Gholami, A. A., Wahid, M. A., Mohammed, H. A. (2014). Heat transfer enhancement and pressure drop for fin-and-tube compact heat exchangers with wavy rectangular winglet-type vortex generators. International Communications in Heat and Mass Transfer, 54, 132-140.

[21] Yilmaz, T., Erdinc, M. T. (2014). Fluid Flow Mixing For Heat Transfer Enhancement In Communicating Converging And Diverging Channels. In Ichmt Digital Library Online. Begel House Inc..

[22] Kwankaomeng, S., Promvonge, P. (2010). Numerical prediction on laminar heat transfer in square duct with 30 angled baffle on one wall. International Communications in Heat and Mass Transfer, 37(7), 857-866.

[23] Li, Z., Gao, Y. (2017). Numerical study of turbulent flow and heat transfer in cross-corrugated triangular ducts with delta-shaped baffles. International Journal of Heat and Mass Transfer, 108, 658-670.

[24] Promvonge, P., Jedsadaratanachai, W., Kwankaomeng, S. (2010). Numerical study of laminar flow and heat transfer in square channel with 30 inline angled baffle turbulators. Applied Thermal Engineering, 30(11-12), 12921303.

[25] Yilmaz, T., Erdinc, M. T. (2014). Fluid Flow Mixing For Heat Transfer Enhancement In Communicating Converging And Diverging Channels. In Ichmt Digital Library Online. Begel House Inc..

[26] Webb, R. L. (1981). Performance evaluation criteria for use of enhanced heat transfer surfaces in heat exchanger design. International Journal of Heat and Mass Transfer, 24(4), 715-726.

[27] Çengel, A. Y. (2006). Heat and Mass Transfer: A Practical Approach, McGraw-Hill, Boston.

[28] Bhuiya, M. M. K., Chowdhury, M. S. U., Saha, M., Islam, M. T. (2013). Heat transfer and friction factor characteristics in turbulent flow through a tube fitted with perforated twisted tape inserts. International Communications in Heat and Mass Transfer, 46, 49-57. 